\title{
RISK FACTORS FOR EARLY RECURRENT LUMBAR DISC HERNIATION: EVALUATION OF 1453 PATIENTS
}

\author{
๑ Burak EREN, ๑ IIlker GÜLEÇ \\ University of Health Sciences Turkey, Bağcılar Training and Research Hospital, Clinic of Neurosurgery, Istanbul, Turkey
}

Objective: Early recurrence of lumbar disc herniation (eRLDH) is a common complication of lumbar disc herniation (LDH) surgery. The aim of this study was to determine the risk factors for eRLDH.

Materials and Methods: Data from 1453 patients who underwent surgery for LDH at our clinic between April 2012 and April 2019 were extracted from the hospital records. Eighty-eight patients underwent repeat surgery due to eRLDH in the postoperative period (between 2 and 12 months). In total, 101 patients who underwent surgery for LDH, but who did not present with eRLDH, were randomly allocated to the control group. The demographic characteristics, types of surgery and magnetic resonance images of the patients were examined.

Results: The mean age of the 189 patients was $46.5 \pm 11.4$ years. Age, gender, occupation and body mass index were not risk factors for eRLDH. However, patients with eRLDH were shorter in height. Smoking status of the patients with LDH was a risk factor for eRLDH. No relationship was found between disc level and eRLDH. eRLDH was less common in patients who underwent aggressive discectomy. In addition, the location of the herniated disc in the axial plane, its migration in the vertical plane and its relationship with the posterior longitudinal ligament were not risk factors for eRLDH. The disc height in the operated level was lower in the eRLDH group.

Conclusion: Being short in height, smoking, limited discectomy and low disc height are risk factors for eRLDH.

Keywords: Early recurrence lumbar disc herniation, recurrent lumbar disc herniation, lumbar disc herniation, LDH

\section{INTRODUCTION}

A common complication of lumbar disc herniation (LDH) surgery is recurrence of the LDH. The rate of recurrence of LDH is between $5 \%$ and $15 \%(1,2)$. In patients with LDH recurrence, a repeat surgery is more difficult due to the presence of epidural fibrosis and scar tissue ${ }^{(3)}$. In addition, functional impairment and increase in treatment costs pose an important problem. Some risk factors for recurrent LDH have been reported, such as limited discectomy and heavy workload ${ }^{(2,4,5)}$; however, the risk factors for early recurrence of lumbar disc herniation (eRLDH) have not been studied previously.

In the present study, we aimed to determine the risk factors for eRLDH in patients who underwent repeat surgery for LDH in the early postoperative period.

\section{MATERIALS AND METHODS}

This retrospective study was approved by the local ethics committee of our hospital (date: 15.02.2019, number: 2019.02.2.02.010.r018).

Data from 1453 patients who underwent surgery for LDH at our clinic between April 2012 and April 2019 was used for this study. These patients underwent single-level microdiscectomy using the unilateral fenestration technique. Ninety-six patients underwent repeat surgery for eRLDH in the postoperative period (2-12 months). Four patients whose records were incomplete and for whom magnetic resonance imaging (MRI) data was not available and four patients who reported that their pain did not subside in the postoperative period, were not included in the study. In addition, patients with a previous history of spinal surgery, bilateral or multilevel LDH, lumbar spinal stenosis, spondylolysis, tumour, trauma, infection, congenital or acquired deformity and instability and patients with eRLDH within 2 months after the first surgery were excluded from the study.

The study group consisted of 88 patients who underwent a repeat surgery for eRLDH. A total of 101 patients who underwent surgery for LDH, but who did not develop eRLDH were randomly allocated to the control group. The patients in the control group were contacted by phone to confirm that they had not undergone surgery for eRLDH and that there were no complaints associated with the disease in the first year after surgery. From the patients' hospital records, age, gender, weight $(\mathrm{kg})$, height $(\mathrm{cm})$, occupation, smoking status, recurrence time (days), the level and side of LDH and the type of surgery (limited 
or radical discectomy) were examined by the same spinal surgeon.

In addition, for the eRLDH and control groups, MRI data from each patient before the first surgery was examined. Specifically, the height of the herniated intervertebral disc (IDH; mm), horizontal placement of the disc (central/paramedian/ foraminal/far/lateral), migration of the disc in the vertical plane (up/middle/down) and the relationship of the disc to the posterior longitudinal ligament (subligamentous/sequestrated) were examined. IDH was calculated as the mean of the herniated disc heights in the sagittal plane of the anterior portion, posterior portion and midpoint.

\section{Statistical Analysis}

Statistical analysis was performed using the Number Cruncher Statistical System 2007 software (Utah, USA) package programme with the help of a statistician. Using descriptive statistical methods (mean, standard deviation) and the ShapiroWilk normality test, the distribution of variables was examined. An independent t-test was used to compare binary groups with a normal distribution. A $p$ value $<0.05$ was considered statistically significant.

\section{RESULTS}

The mean age of the patients $(n=189)$ was $46.5 \pm 11.4$ years (range: 20-70 years). Age was not found to be a risk factor for eRLDH. The median recurrence time after the first surgery was 167 days (range, 60-362 days). A total of 97 patients were male (51.3\%) and 92 were female (48.7\%). Body mass index (BMI) was greater in the group with eRLDH, although there was no statistically significant difference $(p=0.295)$. A statistically significant difference was observed between the mean height of the patients in the two groups; patients in the eRLDH group were shorter ( $p=0.003$ ). Heavy workload (occupation) was not shown to be a risk factor for eRLDH in patients with LDH $(p=0.508)$, while smoking status in patients with LDH was a risk factor for eRLDH ( $p=0.001)$. The demographic data of the eRLDH and control groups is shown in Table 1.

Although eRLDH was more commonly observed at the level of L3-4, there was no relationship between disc level and eRLDH $(p=0.457)$. Overall, there was one case of eRLDH at the level of L1-2, 2 cases at the level of L2-3, 11 cases at the level of L3-4, 44 cases at the level of L4-5 and 30 cases at the level of L5-S1 (Figure 1). There was no relationship between eRLDH and left or right sided LDH surgery $(p=0.54)$. The development of eRLDH was not significantly affected by the location of the herniated disc in the axial plane. There was no relationship between eRLDH and migration of the herniated disc in the vertical plane. Whether the herniated disc was sequestered or subligamentous was also not associated with eRLDH $(p=0.058)$. The mean IDH was lower in the eRLDH group $(p=0.02)$. When correlation analysis was performed, IDH was found to be a risk factor independent of height. In addition, the recurrence rate was significantly lower in patients who underwent aggressive discectomy $(p=0.015)$. MRI data from both groups is shown in Table 2.

Table 1. Demographic data of patients

\begin{tabular}{|c|c|c|c|}
\hline & $\begin{array}{l}\text { eRLDH }(+) \\
(n=88)\end{array}$ & $\begin{array}{l}\text { eRLDH (-) } \\
(n=101)\end{array}$ & p \\
\hline \multirow{2}{*}{ Age } & \multirow{2}{*}{$47.59 \pm 11.3$} & \multirow{2}{*}{$45.67 \pm 11.4$} & 0.250 \\
\hline & & & 0.699 \\
\hline$\leqslant 35$ yrs & 13 (14.8\%) & 15 (14.8\%) & - \\
\hline $36-55$ yrs & 56 (63.6\%) & 69 (68.3\%) & - \\
\hline$>55$ yrs & 19 (21.6\%) & 17 (16.9\%) & - \\
\hline \multicolumn{3}{|l|}{ Gender } & \multirow{3}{*}{0.528} \\
\hline Male & 43 (48.8\%) & 54 (53.4\%) & \\
\hline Female & 45 (51.2\%) & 47 (46.6\%) & \\
\hline Height (cm) & $166.6 \pm 7.1$ & $170.2 \pm 9.1$ & 0.003 \\
\hline Weight (kg) & $80.1 \pm 13$ & $81.8 \pm 8.8$ & 0.297 \\
\hline BMI & $29 \pm 5.5$ & $28.3 \pm 3.4$ & 0.295 \\
\hline \multicolumn{3}{|l|}{ Occupation } & \multirow{3}{*}{0.508} \\
\hline Light & 57 (64.7\%) & 70 (69.3\%) & \\
\hline Heavy & 31 (35.3\%) & 31 (30.7\%) & \\
\hline \multicolumn{3}{|l|}{ Smoking } & \multirow{3}{*}{0.001} \\
\hline Yes & 58 (65.9\%) & 42 (41.5\%) & \\
\hline No & 30 (34.1\%) & 59 (58.5\%) & \\
\hline
\end{tabular}

$\mathrm{n}$ : Number, eRLDH: Early recurrence of lumbar disc herniation, BMI: Body mass index 
Table 2. Comparison of lumbar disc herniation MRI findings in groups

\begin{tabular}{|c|c|c|c|}
\hline & $\begin{array}{l}\text { eRLDH }(+) \\
(n=88)\end{array}$ & $\begin{array}{l}\text { eRLDH }(-) \\
(n=101)\end{array}$ & $\mathbf{p}$ \\
\hline \multicolumn{3}{|l|}{ Side } & \multirow{3}{*}{0.54} \\
\hline Right & 44 (50\%) & 55 (54.5\%) & \\
\hline Left & $44(50 \%)$ & $46(45.5 \%)$ & \\
\hline \multicolumn{3}{|l|}{ Axial Placement } & \multirow{5}{*}{0.679} \\
\hline Far lateral & $2(2.3 \%)$ & 2 (1.9\%) & \\
\hline Foraminal & $17(19.3 \%)$ & $24(23.8 \%)$ & \\
\hline Paramedian & 52 (59.1\%) & $51(50.5 \%)$ & \\
\hline Central & 17 (19.3\%) & $24(23.8 \%)$ & \\
\hline \multicolumn{3}{|l|}{ Vertical Migration } & \multirow{4}{*}{0.216} \\
\hline Downward & $38(43.2 \%)$ & $53(52.5 \%)$ & \\
\hline Disc Level & $32(36.3 \%)$ & $25(24.8 \%)$ & \\
\hline Upward & $18(20.5 \%)$ & $23(22.7 \%)$ & \\
\hline \multicolumn{3}{|c|}{ Relationship with PLL } & \multirow{3}{*}{0.058} \\
\hline Sequestered & $29(32.9 \%)$ & 47 (46.6\%) & \\
\hline Subligamentous & 59 (67.1\%) & 54 (53.4\%) & \\
\hline IDH & $7.6 \pm 1.5$ & $8.3 \pm 1.6$ & 0.02 \\
\hline \multicolumn{3}{|c|}{ Type of Discectomy } & \multirow{3}{*}{0.015} \\
\hline Aggressive & 29 (32.9\%) & $51(50.5 \%)$ & \\
\hline Limited & 59 (67.1\%) & $50(49.5 \%)$ & \\
\hline
\end{tabular}

n: Number, eRLDH: Early recurrence of lumbar disc herniation, PLL: Posterior longitudinal ligament, IDH: Height of the herniated intervertebral disc, MRI: Magnetic resonance imaging

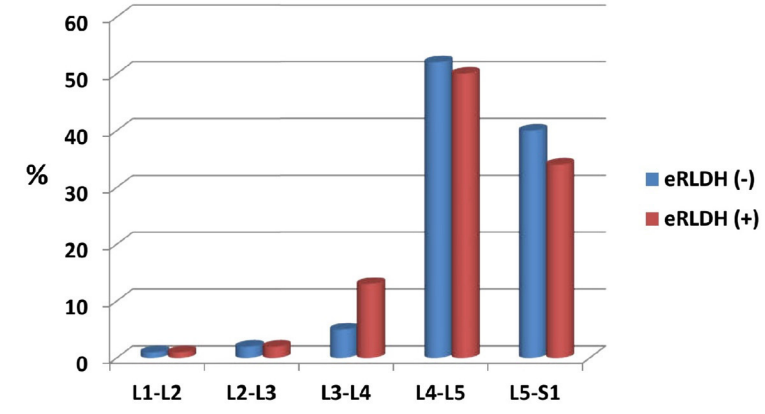

Figure 1. Percentage of patients with and without eRLDH by disc level

eRLDH: Early recurrence of lumbar disc herniation

\section{DISCUSSION}

$\mathrm{LDH}$ is a degenerative disease of the lumbar spine, which frequently occurs in young adults. Recurrence is a common complication of LDH. The patients are often admitted to the hospital complaining of pain and experiencing difficulties in movement ${ }^{(6)}$. Studies have shown that approximately $5-11 \%$ of patients undergo repeat surgery due to recurrence of $\mathrm{LDH}^{(4)}$. Oh et al. ${ }^{(7)}$ considered recurrent LDH that occurred within 6 months as residual, while Leven et al. ${ }^{(1)}$ considered recurrent LDH as residual when it was observed within 6 weeks after the first surgery. In another study, the time restriction was unnecessary and the emergence of complaints after a painless period was considered as recurrent ${ }^{(4)}$. In this study, we considered recurrent LDH occurring before 2 months as residual; thus, we did not include these cases in the study.

Yurac et al. ${ }^{(8)}$ reported that patients under the age of 35 years are at risk of developing recurrent LDH; however, age was not a risk factor for eRLDH in our study. A previous study reported that the rate of recurrence of LDH is higher in males ${ }^{(9)}$, while another study reported that it was higher in females ${ }^{(7)}$. However, Huang et al. ${ }^{(2)}$ did not find gender to be a risk factor in their meta-analysis, which is similar to our study.

Quah et al. ${ }^{(10)}$ reported that obesity is not a predictor of LDH recurrence. In addition, another study reported that BMI is not a risk factor for LDH recurrence ${ }^{(2)}$. In contrast, Meredith et al. ${ }^{(6)}$ showed that obesity is an independent risk factor and reported that the risk of recurrence increases 12 times in patients with a $\mathrm{BMI}>30$. In our study, although a relationship could not be established between BMI and eRLDH, patients with eRLDH were found to be shorter in height. In contrast, Shimia et al. ${ }^{(11)}$ reported that LDH recurrence is more likely to occur in taller patients. In the same study, the rate of recurrence was higher in patients with LDH who were in occupations with a heavy workload. However, in agreement with our study, there are certain studies in the literature that do not support this ${ }^{(2)}$. 
In animal experiments, smoking has been shown to cause constriction of capillaries at the bone-disc junction and dramatically disrupts blood supply to the $\operatorname{disc}^{(12)}$. Numerous studies have revealed a relationship between recurrent LDH and smoking(4). Our study also showed that smoking is a risk factor for eRLDH, which is in agreement with the literature. However, few studies have shown that there is no relationship between smoking and LDH recurrence ${ }^{(7)}$.

It was previously reported that patients with subligamentous disc hernias are at risk of developing recurrent $\mathrm{LDH}^{(8)}$. Miwa et al. ${ }^{(13)}$ studied the relationship between protruded, extruded and sequestrated disc fragments and recurrent LDH. They found that the rate of recurrence was higher in cases of extruded disc hernia. However, another study reported that the presence of a subligamentous disc in the first surgery is an important risk factor for LDH recurrence ${ }^{(9)}$. In our study, the rate of eRLDH occurrence in protruded discs was high. After examining the literature, no relationship was found between LDH recurrence and discectomy level(2). Similarly, we did not identify a relationship between disc level and eRLDH. In addition, the side (right-left) from which the discectomy was performed was not found to be a risk factor for eRLDH.

Leven et al.(1) reported that the rate of paramedian disc hernia was $78 \%$ in patients with recurrent LDH. In our study, this rate was $59.1 \%$ and the placement of the disc in the axial plan was not shown to be a risk factor for eRLDH. This is similar to another study, which did not find this to be a risk factor for recurrent $\mathrm{LDH}^{(14)}$. In a previous study that enrolled 1020 patients, the herniated disc migrated caudally in $72.2 \%$ of patients $^{(15)}$. In our study, this rate was $43.1 \%$ and there was no correlation between disc migration in the vertical plane and the development of eRLDH. Yurac et al. ${ }^{(8)}$ reported that disc migration in the vertical plane is not a risk factor for recurrence, which is in support of our study.

Ledic et al. ${ }^{(16)}$ reported a mean IDH of $8.3 \pm 1.8 \mathrm{~mm}$. Kim et al.(17) reported that the rate of occurrence of recurrent LDH was observed to be higher in the group with a higher IDH. However, we found that eRLDH was more frequent in the group with a low IDH; therefore, low IDH was concluded to be a risk factor for eRLDH.

In the present study, the rate of eRLDH was higher in patients with limited discectomy. McGirt et al. ${ }^{(18)}$ also reported that the most important cause of recurrent LDH is inadequate disk removal. Studies have shown that patients with limited discectomy reported a higher recurrence rate, but less back pain and better clinical improvement were observed in the first year $^{(4,19)}$.

\section{Study Limitations}

This study has the following limitations: disc degeneration of the patients was not evaluated and patients with comorbidities (Diabetes Mellitus, connective tissue diseases, etc.) were excluded from the study.

\section{CONCLUSION}

Risk factors for the development of eRLDH include being short in height, smoking, limited discectomy and low disc height. Gender, age, BMI, occupation, disc level and side, subligamentous or sequestrated discs, the location of the herniated disc in the axial plane and migration of the herniated discs in the vertical planes were not risk factors for the development of eRLDH. Accordingly, if some risk factors can be modified, the rate of eRLDH can be reduced.

\section{Ethics}

Ethics Committee Approval: This retrospective study was approved by the local ethics committee at our hospital (date: 15.02.2019, number: 2019.02.2.02.010.r018).

Informed Consent: No informed consent is required since this study is retrospective.

\section{Authorship Contributions}

Surgical and Medical Practices: B.E., Concept: B.E., I.G., Design: B.E., Data Collection or Processing: B.E., Analysis or Interpretation: I.G., Literature Search: I.G., Writing: B.E.

Conflict of Interest: No conflict of interest was declared by the authors.

Financial Disclosure: The authors declared that this study received no financial support.

\section{REFERENCES}

1. Leven D, Passias PG, Errico TJ, Lafage V, Bianco K, Lee A, et al. Risk Factors for Reoperation in Patients Treated Surgically for Intervertebral Disc Herniation A Subanalysis of Eight-Year SPORT Data. J Bone Joint Surg Am. 2015;97:1316-25.

2. Huang W, Han Z, Liu J, Yu L, Yu X. Risk Factors for Recurrent Lumbar Disc Herniation A Systematic Review and Meta-Analysis. Medicine (Baltimore). 2016;95:e2378.

3. Circi E, Ozalay M, Caylak B, Bacanli D, Derincek A, Tuncay IC. The effect of oophorectomy on epidural fibrosis after laminectomy: an experimental study in rats. Acta Orthop Traumatol Turc. 2013;47:193-200.

4. Shin BJ. Risk Factors for Recurrent Lumbar Disc Herniations. Asian Spine J 2014;8:211-5.

5. Dora C, Schmid MR, Elfering A, Zanetti M, Hodler J, Boos N. Lumbar Disk Herniation: Do MR Imaging Findings Predict Recurrence after Surgical Diskectomy? Radiology. 2005;235:562-7.

6. Meredith DS, Huang RC, Nguyen J, Lyman S. Obesity increases the risk of recurrent herniated nucleus pulposus after lumbar microdiscectomy. Spine J. 2010;10:575-80.

7. Oh JT, Park KS, Jung SS, Chung SY, Kim SM, Park MS, et al. Surgical Results and Risk Factors for Recurrence of Lumbar Disc Herniation. Korean J Spine. 2012;9:170-5.

8. Yurac R, Zamorano JJ, Lira F, Valiente D, Ballesteros V, Urzúa A. Risk factors for the need of surgical treatment of a first recurrent lumbar disc herniation. Eur Spine J. 2016;25:1403-8.

9. Morgan-Hough CV, Jones PW, Eisenstein SM. Primary and revision lumbar discectomy. A 16-year review from one centre. J Bone Joint Surg Br. 2003;85:871-4.

10. Quah C, Syme G, Swamy GN, Nanjayan S, Fowler A, Calthorpe D. Obesity and recurrent intervertebral disc prolapse after lumbar microdiscectomy. Ann R Coll Surg Engl. 2014;96:140-3. 
11. Shimia M, Babaei-Ghazani A, Sadat BE, Habibi B, Habibzadeh A. Risk factors of recurrent lumbar disk herniation. Asian J Neurosurg. 2013;8:93-6.

12. Holm S, Nachemson A. Nutrition of the intervertebral disc: acute effects of cigarette smoking. An experimental animal study. Ups J Med Sci. 1988;93:91-9.

13. Miwa S, Yokogawa A, Kobayashi T, Nishimura T, Igarashi K, Inatani $H$, et al. Risk factors of recurrent lumbar disc herniation: a single center study and review of the literature. I Spinal Disord Tech. 2015;28:E265-9.

14. Häkkinen A, Kiviranta I, Neva MH, Kautiainen $H$, Ylinen J. Reoperations after first lumbar disc herniation surgery; a special interest on residives during a 5-year follow-up. BMC Musculoskelet Disord. 2007;8:2.

15. Daghighi MH, Pouriesa M, Maleki M, Fouladi DF, Pezeshki MZ, Mazaheri Khameneh $\mathrm{R}$, et al. Migration patterns of herniated disc fragments: a study on 1,020 patients with extruded lumbar disc herniation. Spine J. 2014;14:1970-7.
16. Ledic D, Vukas D, Grahovac G, Barth M, Bouma G), Vilendecic M. Effect of Anular Closure on Disk Height Maintenance and Reoperated Recurrent Herniation Following Lumbar Diskectomy: Two-Year Data. J Neurol Surg A Cent Eur Neurosurg. 2015;76:211-8.

17. Kim KT, Park SW, Kim YB. Disc height and segmental motion as risk factors for recurrent lumbar disc herniation. Spine. 2009;34:2674-8.

18. McGirt MJ, Eustacchio S, Varga P, Vilendecic M, Trummer M, Gorensek $M$, et al. A prospective cohort study of close interval computed tomography and magnetic resonance imaging after primary lumbar discectomy: factors associated with recurrent disc herniation and disc height loss. Spine (Phila Pa 1976). 2009;34:2044-51.

19. Carragee EJ, Spinnickie AO, Alamin TF, Paragioudakis S. A prospective controlled study of limited versus subtotal posterior discectomy: short-term outcomes in patients with herniated lumbar intervertebral discs and large posterior anular defect. Spine (Phila Pa 1976). 2006;31:653-7. 